\title{
Síndrome de Möbius: achados clínicos e cirúrgicos em 7 pacientes
}

\author{
Möbius syndrome:clinical and surgical findings in 7 patients
}

\author{
Galton Carvalho Vasconcelos ${ }^{1}$ \\ Frederico Bicalho Dias da Silva ${ }^{2}$ \\ Henderson Celestino de Almeida ${ }^{3}$ \\ Maria de Lourdes M. V. Boas ${ }^{4}$ \\ Miguel Gontijo Álvares ${ }^{5}$
}

\begin{tabular}{|c|}
\hline RESUMO \\
\hline Objetivo: Avaliar o quadro clínico, as anormalidades gestacionais e de \\
parto e os resultados na cirurgia de estrabismo em pacientes com síndrome \\
de Möbius. Métodos: Foram estudados, retrospectivamente, 7 pacientes \\
com diagnóstico de síndrome de Möbius, dos quais 6 foram submetidos \\
à cirurgia de estrabismo. Resultados: Além dos achados clássicos que \\
caracterizam a síndrome, em todos os casos estudados observaram-se \\
alterações gestacionais ou de parto. Em 2 pacientes encontrou-se no per- \\
operatório, inserção posteriorizada do reto medial. Graças à fixação cruza- \\
da, esses pacientes geralmente não desenvolvem ambliopia. Conclusão: \\
A cirurgia, quando indicada, deve constar apenas dos retrocessos mus- \\
culares e acredita-se que o planejamento cirúrgico deva ser menor do que \\
o feito em casos de paralisia isolada do nervo abducente de mesmo valor. \\
Descritores: Síndrome de Möbius/diagnóstico; Estrabismo/cirurgia
\end{tabular}

\section{INTRODUÇÃO}

A síndrome de Möbius é uma condição pouco comum, caracterizada por paralisia de nervos cranianos associada a outras anormalidades, especialmente dos membros. De acordo com Reed e Grant ${ }^{(1)}$, os primeiros casos foram descritos por Von Graefe em 1880, Harlan em 1881 e Chisolm em 1882. Möbius $^{(2-3)}$, em 1888 e 1892 estudou 43 casos de paralisia dos nervos cranianos, identificando um grupo de 6 pacientes que apresentavam paralisia dos nervos faciais e dos abducentes. Desde então, o nome de Möbius tem sido associado a esta condição. Em 1939, Henderson ${ }^{(4)}$ revisou os achados de 61 pacientes, reconhecendo as anormalidades sistêmicas associadas, ampliando desta maneira o espectro desta síndrome.

A etiologia da síndrome de Möbius não é clara, sendo considerada em alguns estudos como resultante de agressão ao feto entre a quarta e quinta semanas da gestação ${ }^{(1)}$. Em alguns casos, porém, parece haver clara evidência de herança autossômica dominante ${ }^{(5)}$. Seja por agressão geneticamente determinada, seja por alterações locais na embriogênese, alguns estudos em animais sugerem a participação de eventos hipóxicos ou isquêmicos cerebrais na etiologia da síndrome de Möbius ${ }^{(6)}$.

As características clínicas mais importantes são: paralisia facial do tipo periférico, geralmente bilateral, paralisia do sexto par craniano, atrofia da musculatura lingual e outras anomalias musculares e ósseas, localizadas com maior freqüência nos membros superiores e inferiores, em sua porção distal. Estas alterações produzem uma "facies" característica, de aspecto inexpressivo devido à ausência de mímica facial. Além disso, a boca mantém-se entreaberta e os olhos não se fecham, mas ao tentar fechá-los 
observa-se o sinal de Bell. As alterações da musculatura oculomotora são variáveis. Na maioria das vezes, existe paralisia dos músculos retos laterais, com esotropia de grande ângulo e rotação da cabeça, de modo a fixar com cada olho em adução. Outras vezes há esotropia discreta, geralmente associada à paralisia conjugada do olhar. Os movimentos verticais do olho são geralmente normais. Outras paralisias de nervos cranianos, principalmente bulbares, podem estar presentes. Alguns recém-nascidos afetados apresentam dificuldade para deglutição, devido à paralisia do nono e décimosegundo nervos cranianos. Retardo mental leve tem sido relatado em vários pacientes ${ }^{(4)}$; entretanto em muitos casos os problemas na fala e a falta de expressão facial conduzem erroneamente ao diagnóstico de retardo mental.

\section{MÉTODOS}

Retrospectivamente, foram estudados 7 pacientes no Serviço de Estrabismo do Hospital São Geraldo: 4 pacientes do sexo masculino e 3 do sexo feminino. A idade em 6 pacientes, na ocasião da cirurgia, variou de 03 a 12 anos (média de 6,30 ). O paciente $n^{\circ} 7$, não operado, apresentava 8 anos ao primeiro exame. Todos os pacientes apresentaram paralisia ou paresia dos nervos abducentes e dos faciais causando, respectivamente, esotropia que variou entre 35 a 85 DP e ausência de expressão facial, além de alterações osteomusculares (Figura 1). Foram anotadas: a acuidade visual em ambos os olhos, a medida do desvio pelo teste de cobertura ou método de Krimsky, as rotações, a biomicroscopia, a retinoscopia sob cicloplegia e a fundoscopia além da pesquisa de anormalidades gestacionais e do parto.

A rotação ocular normal foi expressa pela letra $\mathrm{N}$ e a deficiência de rotação por $-1(25 \%)$ a $-4(100 \%)$. Se à tentativa de abdução, o olho do paciente não chegasse à linha média, expressou-se essa deficiência por -5 (discreta) -6 (moderada)

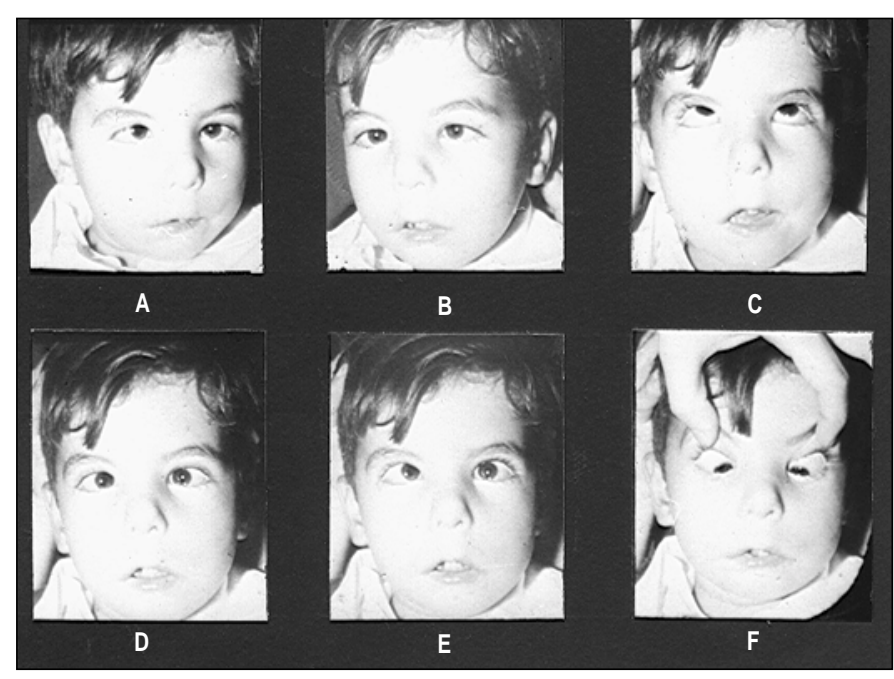

Figura 1 - Síndrome de Möbius. Estrabismo fixo - A: Fixa OE, ET 60DP; B: Fixa OD, ET 60DP; C: Supraversão: Normal; D: Dextroversão: RLD -7, RME -1; E: Levoversão: RLE -7, RMD -1; F: Infraversão: Normal ou -7 (acentuada); se permanecesse em adução máxima (estrabismo fixo) seria classificado como -8 .

Foi considerado um bom resultado cirúrgico um eso ou exodesvio de até 10 dioptrias prismáticas.

\section{RESULTADOS}

Em todos os casos houve problemas gestacionais ou de parto, sendo que a mãe de um deles tomou talidomida durante a gravidez.

Alteração da mímica facial e paresia ou paralisia do nervo abducente ocorreram em todas as crianças. Defeitos das extremidades, distrofia da língua, anormalidades maxilomandibulares e distúrbios da fala apareceram em 4 casos. Houve também atraso em iniciar a deambulação em 3, implantação baixa e saliente do pavilhão auditivo e convulsões em 2, e hérnia inguinal e malformação óssea do tórax em 1 paciente (Tabela 1 ).

Todos os pacientes apresentaram hipermetropia variando $\mathrm{de}+0,50 \mathrm{a}+6,00$ dioptrias, associada ou não a astigmatismo, mostrando um equivalente esférico de $+1,00 \mathrm{a}+4,00$ (média $=$ $+2,7$ ). O paciente $\mathrm{n}^{\circ} 5$ apresentou aniso-hipermetropia (de 2,5 dioptrias). Fixação cruzada foi observada em quatro dos sete casos. A esotropia apareceu em todos os casos, variando de 35 a 85 dioptrias prismáticas (média de 52,9). Somente o paciente $n^{\circ} 5$ apresentou hipertropia, de 15 DP. A limitação de abdução, simétrica em 6 casos, variou de -3 a -7; dois pacientes apresentaram "olhos congelados" em convergência. A adução foi normal em dois pacientes e deficiente em 5, variando de -1 a -3. Os movimentos verticais foram normais (Tabela 2).

A análise per-operatória mostrou inserção posteriorizada do reto medial em 2 pacientes, um deles, apresentando também dupla inserção (Tabela 3).

A dução forçada passiva para abdução foi negativa em 1 paciente e positiva em todos os outros. No paciente $n^{\circ} 60$ teste permaneceu positivo após a desinserção do reto medial direito. Foi encontrada uma segunda inserção deste músculo, que desfeita, tornou a dução passiva finalmente negativa. A restrição mecânica foi maior nos casos com as esotropias de maior ângulo.

O plano cirúrgico consistiu de recuo isolado de retos mediais em 3 pacientes e foi associado em 2 casos à ressecção de um reto lateral e em 1 caso à ressecção de um reto inferior. A amplitude dos retrocessos variou de 4 a $6,5 \mathrm{~mm}$ e das ressecções de 10 a $11 \mathrm{~mm}$ (valores não aconselhados mais). Em 1 paciente foram realizados recuo-ressecão monoculares e apenas um não foi operado (Tabela 3 ). No pós-operatório de 1 semana houve 2 casos de ortotropia (Figura 2), 2 casos de esotropia de $10 \mathrm{DP}$, um deles com microhipertropia à esquerda, 1 caso de esotropia de 20 DP associado a hipertropia direita de 10 DP e 1 paciente apresentou exotropia de 20 DP acompanhada de hipertropia esquerda de 10 DP. Ao final de 3 meses, houve ótimo resultado em 3 pacientes e esotropia residual de 20 DP em 2 casos, dos quais um foi reoperado com bom 
Tabela 1. Características dos pacientes com Síndrome de Möbius

\begin{tabular}{|c|c|c|c|}
\hline № & $\begin{array}{l}\text { Anormalidades relacionadas } \\
\text { à gestação e/ou parto }\end{array}$ & Anormalidades gestacionais e características cl & línicas não oculares \\
\hline 2 & Parto com fórceps, difícil, durando 16 horas. & $\begin{array}{l}\text { Paralisia facial bilateral. } \\
\text { Implantação baixa das orelhas. } \\
\text { Hipotonia muscular. }\end{array}$ & $\begin{array}{l}\text { Distúrbio de linguagem. } \\
\text { Começou a andar com } 2 \text { a. e } \\
\text { a falar com } 3,5 \text { a. }\end{array}$ \\
\hline 4 & Parto com fórceps. Ocorreu tocotraumatismo. & Paresia facial bilateral (principalmente à esquerda). & \\
\hline 5 & $\begin{array}{l}\text { Mãe teve rubéola no } 3^{\circ} \text { mês e leiomioma uterino; } \\
\text { fez uso de talidomida. } \\
\text { Parto normal. }\end{array}$ & $\begin{array}{l}\text { Paralisia facial bilateral. } \\
\text { Malformação da língua. } \\
\text { Facomelia e pés voltados para cima. } \\
\text { Hipoplasia mandibular. } \\
\text { Hipotonia generalizada. }\end{array}$ & Começou a andar com 4 a. \\
\hline 6 & Mãe fez tratamento para engravidar. & $\begin{array}{l}\text { Paralisia facial bilateral. } \\
\text { Hiperelastose dos dedos das mãos. } \\
\text { Hérnia inguinal bilateral. } \\
\text { Pé direito de tamanho reduzido. } \\
\text { Língua mal formada. } \\
\text { Hipoplasia mandibular. }\end{array}$ & $\begin{array}{l}\text { Distúrbio de linguagem. } \\
\text { Convulsões. }\end{array}$ \\
\hline 7 & $\begin{array}{l}\text { Mãe sofreu desmaios durante a gravidez. } \\
\text { Parto normal. }\end{array}$ & $\begin{array}{l}\text { Paresia facial à direita e paralisia à esquerda. } \\
\text { Pavilhão auditivo esq. em abano e com im- } \\
\text { plantação baixa. Marcha de base alargada. } \\
\text { Tórax saliente "peito de pombo". }\end{array}$ & $\begin{array}{l}\text { Retardo mental. } \\
\text { Distúrbio de linguagem. } \\
\text { Convulsões. }\end{array}$ \\
\hline
\end{tabular}

\begin{tabular}{|c|c|c|c|c|c|c|c|c|}
\hline \multirow{3}{*}{ № } & \multirow{3}{*}{$\begin{array}{l}\text { Idade na } \\
\text { cirurgia }\end{array}$} & \multirow{3}{*}{ Sexo } & \multirow{3}{*}{$\begin{array}{c}\text { Refração } \\
\text { sob cicloplegia }\end{array}$} & \multirow{3}{*}{$\begin{array}{l}\text { Última } \\
\text { acuidade } \\
\text { visual }\end{array}$} & \multicolumn{4}{|c|}{ Exame Estrabológico } \\
\hline & & & & & \multicolumn{2}{|c|}{ Medida do desvio } & \multicolumn{2}{|c|}{ Rotações } \\
\hline & & & & & Horizontal & Vertical & OD & OE \\
\hline 1 & 12 & Masc & $\begin{array}{l}\mathrm{OD}=+0,50+1,00 \times 15^{\circ} \\
\mathrm{OE}=+0,50+1,00 \times 10^{\circ}\end{array}$ & não informa & $\mathrm{CT}=\mathrm{ET} 85$ & 0 & ${ }_{-6}^{N} \overbrace{N}^{N}-2$ & ${ }_{-2}^{N}{\underset{N}{N}}^{N}-6$ \\
\hline 2 & 4 anos & Masc & $\begin{array}{l}\mathrm{OD}=+6,00+2,00 \times 180^{\circ} \\
\mathrm{OE}=+6,00+2,00 \times 100^{\circ}\end{array}$ & $\begin{array}{l}\text { (fixação cruzada) } \\
\text { não informa }\end{array}$ & $\mathrm{CT}=\mathrm{ET} 40$ & 0 & $\frac{N N}{-4 \underset{N}{X}-2}$ & $\underset{N}{N}{ }_{-2}^{N}-4$ \\
\hline 3 & 7 anos & Fem & $\begin{array}{l}\mathrm{OD}=+1,75+1,75 \times 75^{\circ} \\
\mathrm{OE}=+1,75+2,50 \times 100^{\circ}\end{array}$ & $\begin{array}{l}O D=20 / 25-2 \text { (linear) } \\
O E=20 / 25-2 \text { (linear) }\end{array}$ & $\mathrm{CT}=\mathrm{ET} 45$ & 0 & $\underset{N}{N{ }_{N}^{N}}$ & ${ }_{N}^{N}{\underset{N}{N}-3}_{n}^{N}$ \\
\hline 4 & 3 anos & Fem & $\begin{array}{l}\mathrm{OD}=+1,50 \\
\mathrm{OE}=+1,50\end{array}$ & $\begin{array}{c}\text { (fixação cruzada) } \\
\text { não reage à oclusão } \\
\text { de cada olho }\end{array}$ & $\mathrm{CT}=\mathrm{ET} 50$ & 0 & $\begin{array}{l}N{ }^{N} \\
-6 X^{N}-2\end{array}$ & ${ }_{N}^{N} \underset{N}{X_{N}^{N}}-6$ \\
\hline 5 & 8 anos & Fem & $\begin{array}{l}\mathrm{OD}=+2,50 \\
\mathrm{OE}=+5,00\end{array}$ & $\begin{array}{l}\text { (fixação cruzada) } \\
\text { não reage à oclusão } \\
\text { de cada olho }\end{array}$ & $\mathrm{CT}=\mathrm{ET} 55$ & HT D/E 15 & 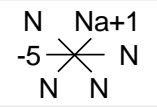 & $\underset{+1}{N \underset{N}{N}-4}$ \\
\hline 6 & 4 anos & Masc & $\begin{array}{l}\mathrm{OD}=+1,00+0,25 \times 75^{\circ} \\
\mathrm{OE}=+2,00+0,25 \times 30^{\circ}\end{array}$ & $\begin{array}{c}\text { (fixação cruzada) } \\
O D=20 / 30-2 \text { (linear) } \\
O E=20 / 40-1 \text { (linear) }\end{array}$ & ET 60 & 0 & $\begin{array}{l}\mathrm{N} N \\
-7 \underset{N}{X}-1\end{array}$ & $\begin{array}{l}N \underset{N}{N}{ }_{-1}^{N}-7 \\
{ }_{N}\end{array}$ \\
\hline 7 & $\begin{array}{c}8 \text { anos } \\
\text { (admissão) } \\
\text { não operado }\end{array}$ & Masc & $\begin{array}{l}\mathrm{OD}=+1,50 \\
\mathrm{OE}=+1,50\end{array}$ & não informa & $\mathrm{KR}=\mathrm{ET} 35$ & 0 & ${ }_{-4}^{N} \overbrace{N}^{N}-3$ & $\begin{array}{l}+1{ }_{N}^{N} \\
-3 \times\end{array}$ \\
\hline
\end{tabular}




\begin{tabular}{|c|c|c|c|c|c|}
\hline № & $\begin{array}{c}\text { Cirurgia feita como } \\
\text { programada }\end{array}$ & $\begin{array}{l}\text { Cirurgia } \\
\text { realizada }\end{array}$ & Reoperação & $\begin{array}{l}\text { Alterações de inserção } \\
\text { do músculo reto }\end{array}$ & $\begin{array}{l}\text { Dução passiva } \\
\text { para abdução }\end{array}$ \\
\hline 1 & $\operatorname{sim}$ & $\begin{array}{l}\text { Recuo RMD } 5 \mathrm{~mm} \\
\text { Recuo RME } 6 \mathrm{~mm}\end{array}$ & não & não & + em AO \\
\hline 2 & $\operatorname{sim}$ & $\begin{array}{l}\text { Recuo RMD } 6 \mathrm{~mm} \\
\text { Recuo RME } 6 \mathrm{~mm}\end{array}$ & não & não & - em AO \\
\hline 3 & $\operatorname{sim}$ & $\begin{array}{l}\text { Recuo RMD } 6 \mathrm{~mm} \\
\text { Recuo RME } 6 \mathrm{~mm}\end{array}$ & não & não & + em AO \\
\hline 4 & $\operatorname{sim}$ & $\begin{array}{l}\text { Ressecção RID } 4 \mathrm{~mm} \\
\text { Recuo RMD } 4 \mathrm{~mm} \\
\text { Recuo RME } 6,5 \mathrm{~mm}\end{array}$ & não & não & + em $\mathrm{AO}$ \\
\hline 5 & $\operatorname{sim}$ & $\begin{array}{c}\text { Recuo RME } 4,5 \mathrm{~mm} \\
\text { Ressecção RLE } 11 \mathrm{~mm}\end{array}$ & $\begin{array}{l}\text { miotomia RMD } 3 / 5 \\
\text { ressecção RLD } 7 \mathrm{~mm}\end{array}$ & RMD a $8 \mathrm{~mm}$ do limbo & +++ em $\mathrm{AO}$ \\
\hline 6 & $\operatorname{sim}$ & $\begin{array}{c}\text { Recuo RMD } 4 \mathrm{~mm} \\
\text { Recuo RME } 5 \mathrm{~mm} \\
\text { Ressecção RLE10mm }\end{array}$ & não & $\begin{array}{l}\text { RMD a } 8 \mathrm{~mm} \text { do limbo } \\
\text { e com inserção dupla } \\
\text { RME a } 7 \mathrm{~mm} \text { do limbo }\end{array}$ & +++ em $\mathrm{AO}$ \\
\hline
\end{tabular}

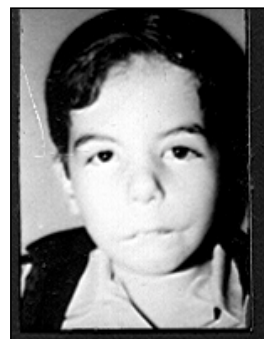

A

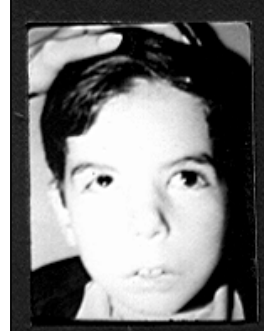

D

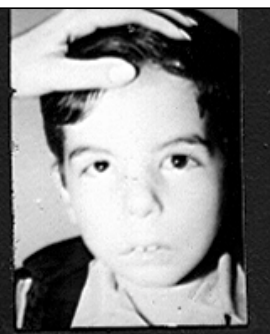

B

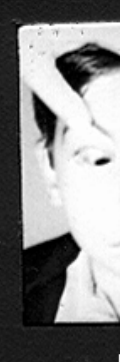

E

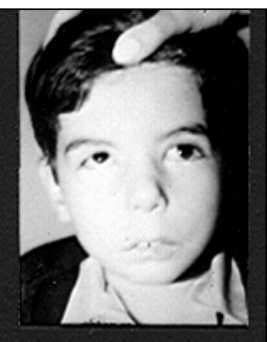

C
Figura 2 - Caso anterior após a cirurgia. A: Orto; B: Dextroversão: RLD -4, RME -3; C: Levoversão: RLE -4, RMD -3; D: Supraversão: OE -2; E: Infraversão: Normal

resultado e o outro não voltou mais ao serviço. Em 1 paciente houve exotropia secundária de $20 \mathrm{DP}$, sendo submetido a 2 aplicações de toxina botulínica ( $5 \mathrm{U}$ em cada reto lateral) com melhora razoável, retornando ao serviço após 1 mês com exotropia de 15 DP (Tabela 4).

\section{DISCUSSÃO}

É interessante enfatizar a presença de anormalidades relacionadas à gestação ou de parto em todos os casos. No caso de $n^{\circ} 5$ a mãe teve rubéola no $3^{\circ}$ mês, leiomioma uterino e tomou talidomida. Papst ${ }^{(7)}$ relatou, entre outros achados, 7 casos de Síndrome de Möbius e 4 casos de Síndrome de
Stilling-Türk-Duane entre os filhos de mães que tomaram talidomida durante a gravidez.

O comprometimento dos nervos abducentes e faciais ocorreu em todos os pacientes com Síndrome de Möbius. Acredita-se que a paralisia dos retos laterais favoreça a contratura dos retos mediais, que gradualmente perdem sua função. A abdução piora com a contratura dos retos mediais, obrigando o paciente a fixar à direita com o $\mathrm{OE}$ e à esquerda com o $\mathrm{OD}, \mathrm{o}$ que provavelmente evita a ambliopia.

Em 2 pacientes notou-se inserção posteriorizada dos retos mediais o que é relatado por Traboulsi e Maumenee ${ }^{(8)}$, que encontraram um reto medial posteriorizado a $20 \mathrm{~mm}$ do limbo e cuja análise anátomo-patológica evidenciou ausência de fibras musculares com presença de apenas tecido fibroso.

\section{CONCLUSÃO}

Os autores acreditam que, realmente os 3 pacientes com melhores resultados cirúrgicos foram aqueles submetidos a retrocesso bimedial. Os pacientes submetidos a retrocessoresecção apresentaram os piores resultados. Devido à grande contratura dos retos mediais, o seu enfraquecimento deve ser menor do que em casos de paralisia isolada de abducentes de mesmo valor. O retrocesso dos retos mediais já deficientes, piora a adução, sendo o resultado ideal, possível, uma microesotropia com olhos congelados horizontalmente. Os movimentos oculares verticais geralmente permanecem normais. A injeção de toxina botulínica aplicada em apenas 1 caso, produziu melhora razoável, mas não parece estar indicada em pacientes com Síndrome de Möbius.

\section{ABSTRACT}

Purpose: To evaluate clinical features, pregnancy and labour abnormalities and the results of strabismus surgery in patients 


\begin{tabular}{|c|c|c|c|c|c|c|}
\hline \multirow[t]{2}{*}{ № } & \multicolumn{4}{|c|}{ Medida do desvio } & \multicolumn{2}{|c|}{ Rotações: último exame } \\
\hline & 1 semana & 2 meses & 3 meses & Último exame & OD & OE \\
\hline 1 & $\begin{array}{l}\text { Orto } \\
\text { Orto' }\end{array}$ & - & $\begin{array}{l}\text { ET } 6 \\
\text { Orto' }\end{array}$ & $\begin{array}{c}\text { ET } 6 \\
\text { Orto' } \\
4 \text { meses }\end{array}$ & $-4{ }_{N}^{N} \succ_{N}^{N}-4$ & ${ }_{-4}^{N} \overbrace{N}^{N}-4$ \\
\hline 2 & 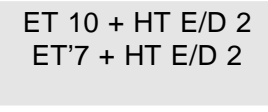 & - & $\begin{array}{l}\text { ET } 5+\text { HT E/D } 3 \\
\text { ET'2 + HT E/D } 2\end{array}$ & $\begin{array}{c}\text { ET } 10 \\
\text { ET'15 } \\
44 \text { meses }\end{array}$ & $-3{ }_{N}^{-1} \chi_{N}^{-1}-1$ & ${ }_{N}^{-1}{ }_{N}^{-2} x_{N}^{-2}-2$ \\
\hline 3 & $\begin{array}{l}\text { Orto } \\
\text { Orto' }\end{array}$ & - & $\begin{array}{l}\text { ET } 10 \\
\text { ET'3 }\end{array}$ & $\begin{array}{c}X(T) 4 \\
X(T)^{\prime} 4 \\
10 \text { meses }\end{array}$ & ${ }^{3}{ }_{N}^{N} \star_{N}^{N}-3$ & $-3{ }_{N}^{N}>_{N}^{N}-3$ \\
\hline 4 & $\begin{array}{l}\text { ET } 20+\mathrm{HT}^{\mathrm{D}} / \mathrm{E} 10 \\
\mathrm{ET}^{\prime} 20+\mathrm{HT} \text { D/E } 10\end{array}$ & $\begin{array}{l}\text { ET } 30+\text { HT D/E } 6 \\
\text { ET'30 + HT D/E } 8\end{array}$ & - & $\begin{array}{l}\text { não retornou } \\
\text { ao serviço }\end{array}$ & ${ }^{-4}{ }_{N}^{N} \chi_{N}^{N}-2$ & ${ }_{-4}^{N} \underset{N}{\chi_{N}^{N}}-4$ \\
\hline 5 & $\begin{array}{l}\text { ET } 10 \\
\text { Orto' }\end{array}$ & - & $\begin{array}{l}\text { ET } 20+\mathrm{HT} \text { D/E } 9 \\
\text { ET' } 15+\mathrm{HT} \text { D/E } 9 \\
\text { reoperação }\end{array}$ & $\begin{array}{l}\mathrm{HT} \text { D/E } 10 \\
\mathrm{HT} \text { D/E } 10 \\
6 \text { meses }\end{array}$ & ${ }_{N}^{N}>_{N}^{N}-3$ & $-3{ }_{N}^{-2}>_{N}^{-2}-4$ \\
\hline 6 & $\begin{array}{l}\text { XT } 20+\text { HT E/D } 10 \\
\text { XT'35 + HT'E/D } 10\end{array}$ & $\begin{array}{l}\text { toxina } \\
\text { botulínica }\end{array}$ & - & $\begin{array}{c}\text { XT15+HTE/D6 } \\
\text { XT6+HTE/D10 } \\
12 \text { meses }\end{array}$ & ${ }_{N}^{N} \chi_{N}^{-1} 3$ & ${ }_{-5}^{N}{ }_{N}^{N} X^{N}-4$ \\
\hline
\end{tabular}

with Möbius syndrome. Methods: The authors studied retrospectively 7 patients with Möbius syndrome, of whom 6 were submitted to strabismus surgery. Results: In addition to the classical findings that characterize the syndrome, pregnancy and labour insults were observed in all cases. In 2 patients a posterior medial rectus insertion was found during surgery. Due to cross-fixation, these patients usually do not develop amblyopia. Conclusions: Surgery should consist of muscle recessions, but recessing them less than it would be done for similar isolated abducens palsy.

Keywords: Möbius syndrome/diagnosis; Strabismus/surgery

\section{REFERÊNCIAS}

1. Reed H, Grant W. Möbius Syndrome. Br J Ophthalmol 1957;41:731-40

2. Möbius PJ. Über angeborene doppelseitige Abducens-facialis lahmung. München Med Wschr 1888;35:91-4.

3. Möbius PJ. Über infantilian kernschwund. München Med Wschr 1892;39:41-5.

4. Henderson JL. The congenital facial diplegia Syndrome: clinical features, pathology and aetiology. A review of sixty-one cases. Brain 1939;62:381-403.

5. Taylor D, Gregson R. Möbius syndrome. In: Good W, Hoyt C, editors. Strabismus management. Massachutess: Butterworth-Heinemann; 1996. p. 77-83.

6. Ghabrial R, Versace P, Kourt G, Lipson A, Martin F. Möbius Syndrome: features and etiology. J Pediatr Opththalmol Strabismus 1998;35:304-11.

7. Papst W. Thalidomid und knogenitale anomalie der augen. Ber Deutsch Ophthalmol Ges 1963;65:209-15.

8. Traboulsi El, Maumenee IH. Extraocular muscle aplasia in Moebius Syndrome. J Pediatr Ophthalmol Strabismus 1986;23:120-2.

\title{
Novidades na Internet!!!
}

\author{
Agora no site CBO você tem disponível todas as informações na íntegra dos \\ Arquivos Brasileiros de Oftalmologia \\ http://www.cbo.com.br/abo
}

